\title{
Microfluidic Approaches for Improved Bioremediation: Monitoring Pollutant Uptake in a Soil Fungus
}

\author{
Claire Baranger ${ }^{1,2}$, Laura Creusot ${ }^{1}$, Xue Sun $^{1}$, Isabelle Pezron ${ }^{1}$, Anne Le Goff ${ }^{2}$, Antoine Fayeulle ${ }^{1}$ \\ ${ }^{1}$ Sorbonne Universités, Université de Technologie de Compiègne, EA4297 TIMR \\ rue du Dr Schweizer, Compiègne, France \\ claire.baranger@utc.fr; antoine.fayeulle@utc.fr \\ ${ }^{2}$ Sorbonne Universités, Université de Technologie de Compiègne, CNRS, UMR 7338 \\ rue du Dr Schweizer, Compiègne, France
}

\section{Extended Abstract}

Polycyclic aromatic hydrocarbons (PAHs) are widespread pollutants raising public health concerns because of their chronic toxicity, and environmental problems due to their persistence and accumulation in the ecosystems. Compared to physical and chemical PAH remediation techniques, bioremediation appears as relatively cost-effective and less detrimental to the soil structure and biological functions.

Some filamentous soil fungi have been shown to degrade organic pollutants, including high-molecular weight PAHs [1], [2]. However, PAHs are hydrophobic compounds with a very high chemical stability, and their low bioavailability is one of the major limitations for bioremediation [3]. Thus the mechanisms of transport and uptake of hydrophobic pollutants in fungi have to be further investigated. The high influence of soil physicochemical properties on bioremediation efficiency on field was also reported as a limitation to the development of this type of techniques [4]. In order to adapt bioremediation protocols to each site's characteristics, we develop a laboratory-scale screening tool allowing for fine control of physicochemical parameters and the observation of pollutant incorporation at the cell scale.

In this project, microfluidic chambers spiked with benzo[a]pyrene (BaP) are used to mimic polluted soil microenvironments. The model organism is a Talaromyces helicus strain previously isolated from contaminated industrial soil and already characterized for its good $\mathrm{BaP}$ degradation abilities.

Biodegradation rates were quantified by solvent extraction and HPLC analysis at a macroscopic scale, both in liquid culture media and industrial soil samples, to establish correlation with microscopic observations. Additionally, different conditions likely to affect degradation rates such as the addition of surfactants, hydrogen peroxide, and co-cultures with bacteria were tested in batch cultures and soil microcosms. We show for instance that degradation rates above $30 \%$ are achieved in liquid cultures, which is consistent with previous results obtained with this species [5]. First results show an increase of degradation rates in conditions of nitrogen depletion, whereas they appear to be unaffected by the addition of commercial surfactant agents.

Microfluidic systems were designed to enable the culture and in vivo observation of mycelium through time-lapse epifluorescence microscopy, and a compartmentalization of pollutant source and inoculum. Intracellular storage of $\mathrm{BaP}$ was confirmed by the observation of vesicles marked with fluorescence after $24 \mathrm{~h}$ incubation with the pollutant. The goal is to identify biostimulation parameters improving benzo[a]pyrene mobilization by the fungus in microsystems. In a later phase of the project, the predictivity of the developed device shall be verified by testing the conditions previously identified as favorable in real contaminated soils at a laboratory scale. A more long-term objective is to develop new site-fitted bioremediation protocols and to confirm their efficiency on field within collaborations with industrial partners.

\section{References}

[1] E. Marco-Urrea, I. García-Romera, E. Aranda, "Potential of non-ligninolytic fungi in bioremediation of chlorinated and polycyclic aromatic hydrocarbons," New Biotechnology, vol. 32, no. 6, p. 620-628, 2015.

[2] M. C. Romero, M. I. Urrutia, H. E. Reinoso, M. M. Kiernan, "Benzo[a]pyrene degradation by soil filamentous fungi," JYFR, vol. 1, no. 2, p. 025-029, 2010. 
[3] J. J. Ortega-Calvo, M. C. Tejeda-Agredano, C. Jimenez-Sanchez, E. Congiu, R. Sungthong, J. L. Niqui-Arroyo and M. Cantos, "Is it possible to increase bioavailability but not environmental risk of PAHs in bioremediation?," Journal of Hazardous Materials, vol. 261, p. 733-745, 2013.

[4] S. Kuppusamy, P. Thavamani, K. Venkateswarlu, Y. B. Lee, R. Naidu, and M. Megharaj, "Remediation approaches for polycyclic aromatic hydrocarbons (PAHs) contaminated soils: Technological constraints, emerging trends and future directions," Chemosphere, 2016.

[5] A. Fayeulle, "Etude des mécanismes intervenant dans la biodégradation des Hydrocarbures Aromatiques Polycycliques par les champignons saprotrophes telluriques en vue d'applications en bioremédiation fongique de sols pollués," Université du Littoral Côte d'Opale, Dunkerque, 2013. 